\title{
ESTUDO DA CINÉTICA E MODELAGEM DA ADSORÇÃO DE ÁCIDOS GRAXOS LIVRES PRESENTES EM BIOCOMBUSTIIVEIS GERADOS POR CRAQUEAMENTO TERMOCATALÍTICO POR ALUMINA ATIVADA
}

\author{
I. V. CURCINO ${ }^{1}$,A. A. MANCIO ${ }^{2}$, K. M. B. da $\mathrm{COSTA}^{3}$, N. T. MACHADO ${ }^{4}$ \\ ${ }^{1}$ Universidade Federal do Pará, Discente da Faculdade de Engenharia Química \\ ${ }^{2}$ Universidade Federal do Pará, Discente do Programa de Pós-Graduação em Engenharia de \\ Recursos Naturais da Amazônia (PRODERNA) \\ ${ }^{3}$ Universidade Federal do Pará, Discente do Programa de Pós-Praduação em Engenharia \\ Química (PPEQ) \\ ${ }^{4}$ Universidade Federal do Pará, Docente da Faculdade de Engenharia Química \\ E-mail para contato: ingrid.curcino@hotmail.com
}

RESUMO - A cinética de adsorção de ácidos graxos livres (AGLs) presentes em frações destiladas (faixa de temperatura de ebulição de $305-400^{\circ} \mathrm{C}$ ) provenientes do Produto Líquido Orgânico (PLO) obtido por craqueamento termocatalítico de óleo vegetal foi investigada pela determinação da variação da concentração de AGLs nos biocombustíveis antes e depois dos experimentos de adsorção com 5\% em massa de alumina ativada termicamente. A capacidade da alumina ativada como adsorvente em relação à adsorção de ácidos graxos livres foi avaliada através de estudos cinéticos em sistema de batelada à temperatura de $26^{\circ} \mathrm{C}$. Os resultados experimentais indicam que a concentração de AGLs presentes nos biocombustíveis foi reduzida após a adsorção com a alumina ativada e foi observado que a taxa inicial de adsorção aumenta com o incremento de AGLs na alimentação assim como o tempo necessário para que o equilíbrio cinético seja atingido. Os modelos cinéticos de pseudo-primeira-ordem e pseudo-segunda-ordem foram aplicados aos dados experimentais e a cinética de pseudosegunda-ordem representou melhor os dados experimentais. Portanto, os resultados mostraram que a alumina ativada termicamente na proporção usada neste trabalho é um adsorvente promissor na remoção dos ácidos graxos livres e que o teor de AGLs tem efeito direto sobre o desempenho da adsorção dos mesmos.

\section{INTRODUÇÃO}

O craqueamento catalítico de óleos vegetais envolve a pirólise na presença de catalisador sólido o que pode melhorar o rendimento dos produtos (TAUFIQURRAHMI e BHATIA, 2011). O aquecimento das moléculas de óleos ou gorduras provoca a quebra das cadeias carbônicas resultando numa mistura de hidrocarbonetos e alguns compostos oxigenados, como ácidos carboxílicos, aldeídos, cetenos e acroleína (QUIRINO, 2006).

Um dos problemas da aplicação direta do PLO é a elevada acidez que este produto apresenta aonde o principal responsável por essa acidez são os ácidos graxos livres (AGLs) 
presentes no produto obtido. Vários processos podem ser empregados com o objetivo de diminuir essa acidez, entre eles o processo de adsorção (HANN,2008).

A adsorção é uma operação de transferência de massa do tipo sólido-fluido na qual se explora a habilidade de certos sólidos em concentrar em sua superfície determinadas substâncias existentes em soluções líquidas ou gasosas, o que permite separá-las dos demais componentes dessas soluções (GOMIDE, 1988).

A alumina sem tratamento não apresenta as características necessárias para um bom adsorvente. Porém, quando ativada termicamente apresenta um amento no número de poros e área superficial específica, o que favorece seu desempenho no processo de adsorção (OLIVEIRA, 2005).

Os estudos sobre a cinética de adsorção são importantes na determinação do tempo necessário para alcançar o equilíbrio, no exame das velocidades de adsorção que podem ser usadas para desenvolver modelos e no entendimento dos processos que influenciam a remoção dos solutos. (FUNGARO e IZIDORO, 2008 ).

Portanto, neste trabalho estudou-se a cinética de adsorção dos AGLs presentes nas frações destiladas na faixa de temperatura de $305-400^{\circ} \mathrm{C}$ provenientes do PLO produzido por craqueamento termocatalítico de óleo de palma bruto, empregando alumina ativada termicamente como adsorvente. Verificou-se o efeito da concentração inicial de AGLs sobre a adsorção e ajustou-se os modelos cinéticos de pseudo-primeira-ordem e pseudo-segundaordem para representar os dados experimentais.

\section{MATERIAIS E MÉTODOS}

\subsection{ADSORVENTE}

$\mathrm{O}$ adsorvente empregado neste trabalho foi a alumina ativada, a qual foi obtida a partir de Hidróxido de Alumínio $\left[\mathrm{Al}(\mathrm{OH})_{3}\right]$, material cedido pela empresa ALUNORTE S/A (Barcarena-Pará-Brasil). Baseado no método descrito por Cunha et al.(2012), as amostras de Hidróxido de Alumínio $\left[\mathrm{Al}(\mathrm{OH})_{3}\right]$ foram lavadas em água $\left(100{ }^{\circ} \mathrm{C}\right)$, sob agitação em béquer, durante 60 minutos, a fim de remover impurezas indesejáveis. Posteriormente, as amostras de $\mathrm{Al}(\mathrm{OH})_{3}$ úmidas foram submetidas a secagem a $105^{\circ} \mathrm{C}$, durante 24 horas, em estufa de recirculação de ar. Após o período de secagem as amostras foram submetidas ao tratamento térmico, o qual foi realizado a $450^{\circ} \mathrm{C}$, em forno elétrico, com controle digital de temperatura, rampa e taxa de aquecimento $\left(5^{\circ} \mathrm{C} / \mathrm{min}\right)$, durante 120 minutos.

\subsection{FRAÇÃO DESTILADA}

Três diferentes frações destiladas na faixa de temperatura de $305-400^{\circ} \mathrm{C}$, apresentadas na Tabela 1, e obtidas no Laboratório de Processo e Separações Térmicas (THERMTEK/FEQ/ITEC/UFPA) através da destilação a pressão atmosférica (coluna de destilação piloto) do PLO foram utilizadas como alimentação a fim de avaliar o efeito do teor de ácidos graxos livres iniciais sobre a adsorção dos mesmos. 
Tabela 1 - Número de ácidos totais (NAT) das frações destiladas destinadas a desacidificação por adsorção

\begin{tabular}{cc}
\hline Alimentação & NAT \\
\hline A & 1,55 \\
B & 17,35 \\
C & 19,65 \\
\hline
\end{tabular}

\subsection{CINÉTICA DE ADSORÇÃO DE ÁCIDOS GRAXOS LIVRES}

Para a obtenção das cinéticas de adsorção dos ácidos graxos livres foram realizados experimentos no sistema de batelada em frascos elernmeyers de $250 \mathrm{~mL}$, os quais foram dispostos em uma mesa agitadora (AGIF/RES-MCT-010/Ética/Q-842-210) com agitação orbital para que as frações destiladas tivessem o contato adequado com o adsorvente. Aproximadamente cinco gramas de fração destilada e $5 \%(\mathrm{~m} / \mathrm{m})$ de adsorvente (alumina ativada termicamente) foram adicionados em cada erlenmeyer e, em seguida, agitou-se por tempos pré-estabelecidos. As seguintes variáveis de processo foram mantidas constantes: temperatura (ambiente), pressão (atmosférica), velocidade de agitação (160 rpm), porcentagem de adsorvente (5\%). Variando apenas o teor de ácidos graxos livres iniciais e o tempo de contato $(0 ; 2,5 ; 5 ; 10 ; 20 ; 30 ; 40 ; 50$ e $60 \mathrm{~min})$.

Após a adsorção, o conteúdo do frasco foi submetido à filtração a vácuo, empregando papel filtro Whatman $\mathrm{n}^{\circ}$ 1, obtendo-se como parte filtrada uma fração destilada desacidificada, a qual foi determinada o NAT de acordo com o método padrão da ASTM D 974.

A quantidade relativa de ácidos graxos livres adsorvidos por grama de adsorvente foi calculada através da Equação 1. A qual representa a quantidade de $\mathrm{KOH}$, em mg, necessária para neutralizar os AGLs adsorvidos em $1 \mathrm{~g}$ de adsorvente.

$$
q_{t}=\frac{\left[\left(N A T_{i}-N A T_{f}\right) \times F D\right]}{A D S}
$$

\subsection{MODELOS CINÉTICOS}

A fim de avaliar um modelo cinético apropriado para representar o experimento, utilizou-se os modelos cinéticos de pseudo-primeira-ordem e pseudo-segunda-ordem de Lagergren, conforme Equações 2 e 3.

$$
\begin{aligned}
& \log _{10}\left(q_{e}-q_{t}\right)=\log _{10}\left(q_{e}\right)-\frac{K_{1} t}{2,303} \\
& \frac{t}{q_{t}}=\frac{1}{K_{2} q_{e}^{2}}+\frac{1}{q_{e}} t
\end{aligned}
$$


Através do valor obtido para $\mathrm{K}_{2}$, a partir da Equação 3, é possível calcular a velocidade de adsorção inicial $\left(\mathrm{h}_{\mathrm{i}}\right)$ para $\mathrm{t}_{0}$, utilizando a Equação 4.

$$
h=k_{2} q_{e}^{2}
$$

\section{RESULTADOS E DISCUSSÃO}

\subsection{Cinética de adsorção e efeito do teor inicial de AGLs}

A quantidade relativa de AGLs adsorvidos sobre a alumina ativada $\left(\mathrm{q}_{\mathrm{t}}\right)$ foi obtida através da Equação 1. O efeito do tempo de contato das frações destiladas com a alumina ativada é apresentado na figura 1 , na forma de gráfico.

Figura 1 - Cinética de adsorção de AGLs de fração destilada na faixa de $305-400{ }^{\circ} \mathrm{C}$

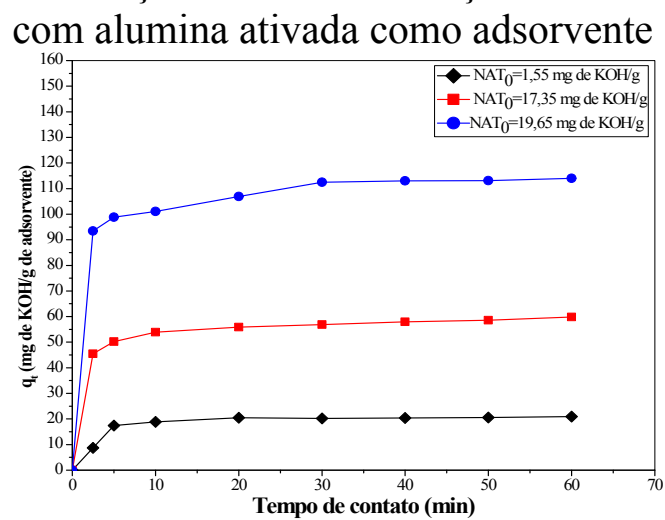

Através da Figura 1, observa-se que a quantidade relativa de AGLs adsorvidos aumenta com o tempo de contato, atingindo uma quantidade máxima e após um determinado período de tempo verifica-se que $\mathrm{q}_{\mathrm{t}}$ se mantém constante para as alimentações $\mathrm{A}, \mathrm{B}$ e $\mathrm{C}$, indicando que o equilíbrio de adsorção foi alcançado. Para a amostra $\mathrm{A}$, o tempo de contato necessário para alcançar o equilíbrio de adsorção dos AGLs presentes na fração destilada foi de 20 min. Em relação às amostras $\mathrm{B}$ e $\mathrm{C}$, o tempo de contato requerido foi de $30 \mathrm{~min}$. Sendo assim, as amostras $\mathrm{B}$ e $\mathrm{C}$ necessitaram um tempo de contato maior parar atingir o equilíbrio de adsorção em relação à amostra $\mathrm{A}$.

\subsection{Modelagem Cinética}

A Figura 2 apresenta o ajuste dos modelos cinéticos aos dados experimentais de adsorção.

Figura 2 - Comparação dos modelos cinéticos da adsorção de AGLs sobre Alumina ativada termicamente: (a) Modelo cinético de Pseudo primeira-ordem; (b) Modelo cinético de

(a)

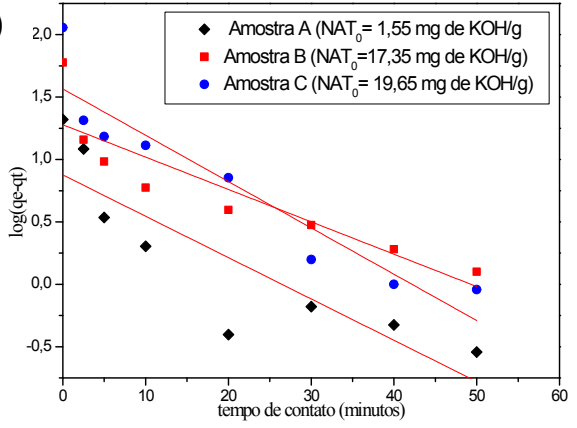

Pseudo-segunda-ordem

(b)

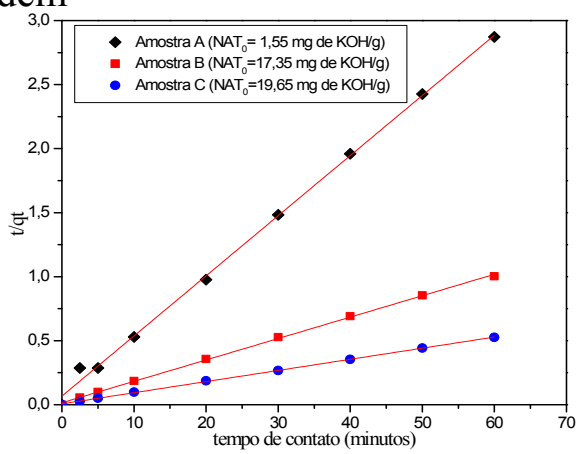


Os parâmetros obtidos a partir das correlações apresentadas na Figura 2 estão contidos na Tabela 3.

Tabela 3 - Parâmetros cinéticos de adsorção referentes aos modelos de pseudo-primeiraordem e pseudo-segunda-ordem para diferentes concentrações iniciais de AGLs

\begin{tabular}{|c|c|c|c|c|c|}
\hline \multirow{2}{*}{$\begin{array}{c}\text { NAT } \\
(\mathrm{mg} \mathrm{de} \mathrm{KOH} / \mathrm{g})\end{array}$} & \multicolumn{5}{|c|}{ Pseudo-primeira-ordem } \\
\hline & $\begin{array}{l}\mathrm{q}_{\mathrm{e}}(\exp ) \\
(\mathrm{mg} / \mathrm{g})\end{array}$ & $\mathrm{K}_{1}\left(\mathrm{~min}^{-1}\right)$ & \multicolumn{2}{|c|}{$\begin{array}{l}\mathrm{q}_{\mathrm{e}}(\text { calc }) \\
(\mathrm{mg} / \mathrm{g})\end{array}$} & $\mathrm{R}_{1}^{2}$ \\
\hline 1,55 & 20,8009 & 0,0763 & \multicolumn{2}{|c|}{7,5206} & 0,7240 \\
\hline 17,35 & 59,8122 & 0,0597 & \multicolumn{2}{|c|}{18,9841} & 0,7790 \\
\hline 19,65 & 114,0374 & 0,0855 & \multicolumn{2}{|c|}{36,7604} & 0,8630 \\
\hline NAT & \multicolumn{5}{|c|}{ Pseudo-segunda-ordem } \\
\hline$(\mathrm{mg}$ de $\mathrm{KOH} / \mathrm{g})$ & $\begin{array}{l}\mathrm{q}_{\mathrm{e}}(\mathrm{exp}) \\
(\mathrm{mg} / \mathrm{g})\end{array}$ & $\mathrm{K}_{2}\left(\min ^{-1}\right)$ & $\begin{array}{l}\mathrm{q}_{\mathrm{e}}(\mathrm{calc}) \\
(\mathrm{mg} / \mathrm{g})\end{array}$ & $\mathrm{h}_{\mathrm{i}}(\mathrm{mg} / \mathrm{g} \cdot \min )$ & $\mathrm{R}_{2}^{2}$ \\
\hline 1,55 & 20,8909 & 0,0329 & 21,2902 & 14,9120 & 0,9978 \\
\hline 17,35 & 59,8122 & 0,0184 & 59,8086 & 65,7030 & 0,9992 \\
\hline 19,65 & 114,0375 & 0,0112 & 114,9425 & 148,3680 & 0,9995 \\
\hline
\end{tabular}

Para verificar qual o modelo cinético que melhor ajustou-se aos dados experimentais, comparou-se quantitativamente os coeficientes de correlação $\left(R^{2}\right)$. Os valores de $R_{2}^{2}$ foram maiores que os valores obtidos para $\mathrm{R}_{1}{ }^{2}$, a partir desta análise confirmou-se que o modelo cinético de pseudo-segunda-ordem representou satisfatoriamente os dados experimentais. Tal afirmação é corroborada pela proximidade dos valores de $\mathrm{q}_{\mathrm{e}}$ determinados experimentalmente e os valores de $\mathrm{q}_{\mathrm{e}}$ calculados a partir do modelo.

Em relação aos valores obtidos para a velocidade inicial de adsorção $\left(\mathrm{h}_{\mathrm{i}}\right)$, observou-se que o incremento no teor inicial de AGLs provoca o aumento da velocidade inicial de adsorção para este experimento.

\section{CONCLUSÃO}

Um incremento na quantidade inicial de ácidos graxos livres na alimentação provocou um aumento no tempo necessário para que o equilíbrio de adsorção seja atingido. Contudo, o aumento do número de ácidos graxos livres iniciais na alimentação proporciona maiores taxas iniciais de adsorção.

Em relação à modelagem cinética, o modelo que representou melhor os dados experimentais foi o modelo cinético de pseudo-segunda-ordem para todas as amostras utilizadas neste experimento.

\section{NOMENCLATURA}
ADS - Massa de adsorvente (g);
FD - Massa de amostra de fração destilada (g);
$\mathrm{h}_{\mathrm{i}}$ - Velocidade de adsorção inicial para $\mathrm{t}_{0}(\mathrm{mg} / \mathrm{g} \min )$; 
$\mathrm{K}_{1}$ - Constante de velocidade de pseudo primeira-ordem $\left(\mathrm{min}^{-1}\right)$;

$\mathrm{K}_{2}$ - Constante de velocidade de pseudo segunda-ordem ( $\mathrm{g} / \mathrm{mg} \mathrm{min}$ );

NAT - Número de ácidos totais (mg de $\mathrm{KOH} / \mathrm{g}$ de amostra);

$\mathrm{q}_{\mathrm{e}}$ - Quantidade de AGLs adsorvida no equilíbrio (mg/g);

$\mathrm{q}_{\mathrm{t}}$ - Quantidade de AGLs adsorvida no tempo $\mathrm{t}(\mathrm{mg} / \mathrm{g})$.

$\mathrm{R}_{1}^{2}$ - Coeficiente de correlação do modelo de pseudo-primeira-ordem;

$\mathrm{R}_{2}{ }^{2}$ - Coeficiente de correlação do modelo de pseudo-segunda-ordem.

\section{REFERÊNCIAS}

AMARAL, A. R. Estudo cinético e das isotermas de adsorção dos óleos de palma (elaies guineenses, jacq.) E andiroba (carapa guianensis, aubl.) Em $\gamma$-alumina e modelagem da dessorção com dióxido de carbono supercrítico. 2013. 145 f. Dissertação (Mestrado em Engenharia Química). Universidade Federal do Pará, Belém, 2013.

FUNGARO, D. A.; IZIDORO, J. C.Modelagem cinética da remoção de $\mathrm{Cd}^{2+}$ por zeólitas de cinzas de carvão. Revista brasileira de ciências ambientais. V.16, pag. 9-13, 2008.

GOMIDE, R. Operações unitárias: operações de transferência de massa. $1^{\text {a }}$ Ed. São Paulo: Dag Gráfica e Editora ltda., 1988 v.4.

HANN, C. J. The Adsorption of Fatty Acids Using Metal Silica Complexes From Rice Husk Ash. 2008. Dissertação de Mestrado. University Sains Malaysia, 2008.

OLIVEIRA, L. B.; ANGÉliCA, R. S.; NECES, R. F.; FIGUEIREDO, B. R. Avaliação de adsorção de íon As (V) em solução sobre alumina ativada, Rejeitos de caulim e SUS produtos de transformação. Workshop Internacional em geologia médica, 2005.

QUIRINO, R.L. Estudo do efeito da presença de alumina dopada com $\mathrm{TiO}_{2}$ e $\mathrm{ZrO}_{2}$ no craqueamento do óleo de soja. 2006. 67 f. Dissertação (Mestrado em Química) Universidade de Brasília, Brasília, 2006.

TAUFIQURRAHMI, N.; BHATIA, S. Catalytic cracking of edible and non-edible oils for the production of biofuels. Energy Environ. Sci. V. 4, pag. 1087-1112, 2011. 